\title{
Scientism recognizes evidence only of the quantitative/general variety
}

\author{
Charles J. Kowalski Ph.D ${ }^{1}$ (1) | Adam J. Mrdjenovich Ph.D ${ }^{2}$ ( ) | Richard W. Redman Ph.D ${ }^{3}$
}

${ }^{1}$ Health Sciences and Behavioral Sciences Institutional Review Board, The University of Michigan, Ann Arbor, Michigan

${ }^{2}$ Office of Research, The University of Michigan, Ann Arbor, Michigan

${ }^{3}$ School of Nursing, The University of Michigan, Ann Arbor, Michigan

\section{Correspondence}

Charles J. Kowalski, Health Sciences and Behavioral Sciences Institutional Review Board, University of Michigan, North Campus Research Complex, 2800 Plymouth Road, Building 520, Suite 1169, Ann Arbor, MI 48109.

Email: chuckk@umich.edu

\begin{abstract}
Rationale, aims and objectives: McHugh and Walker introduced a model of knowledge to demonstrate that EBM is a form of scientism that ignores important sources of knowledge thereby impairing the practice of medicine. We study the development of this model and explore additional applications.

Methods: Review of the relevant literature and identification of possible areas for fruitful application.

Results: We show that the McHugh and Walker model is closely related to the model of evidence considered earlier by Upshur et al. We also indicate that the utility of this model is not limited to showing scientism distorts clinical practice. Several representative applications are identified, including psychotherapy, the Salk polio vaccine trial, and the placebo effect.

Conclusions: Priority should be given to Upshur et al for the development of a model that has far-reaching application to medical epistemology. It is shown that all four of the types of evidence considered-qualitative/personal, qualitative/general, quantitative/general, and quantitative/personal-are required to adequately characterize epistemology in medical research and practice.
\end{abstract}

\section{KEYWORDS}

EBM, efficacy and effectiveness, horses for courses, quantomania, tacit knowledge

\section{1 | INTRODUCTION}

A recent paper in Bioethical Inquiry ${ }^{1}$ makes the interesting and important point that the intrusion of scientism (read EBM) into clinical practice distorts "clinical reality" by ignoring tacit knowledge and thus impairs medical practice. They base their argument on the view that knowledge can be described along two intersecting "dimensions," the tacit-explicit and the particular-general.

The tacit-explicit dimension of knowledge represents the varying degrees to which knowledge can be articulated. Explicit knowledge is that which can be easily articulated, for example, current systolic BP of 149; tacit knowledge cannot be easily (if at all) articulated, for example, how to identify and characterize heart murmurs. Tacit knowledge was first characterized by Polyani, ${ }^{2}$ and its importance in clinical medicine has been recognized by many, see for example, Henry ${ }^{3,4}$ and the references he provides.

The particular-general dimension refers to the degree of applicability of the knowledge: knowledge is "general" to the extent that it can be applied to a group of some kind; it is "particular" to the extent that it is specific to a single individual/thing.

The four kinds of knowledge considered by McHugh and Walker can then be set out as shown in Figure 1 (hereafter referred to as the MW model).

They argue that all four types of knowledge are needed in medicine, and that this scheme "allows us to recognize the different roles and grounding that medical knowledge can have, without presuming any one to be superior or inferior to others" (p. 585). This stands in sharp contrast to the EBM paradigm that focuses exclusively on Q3 


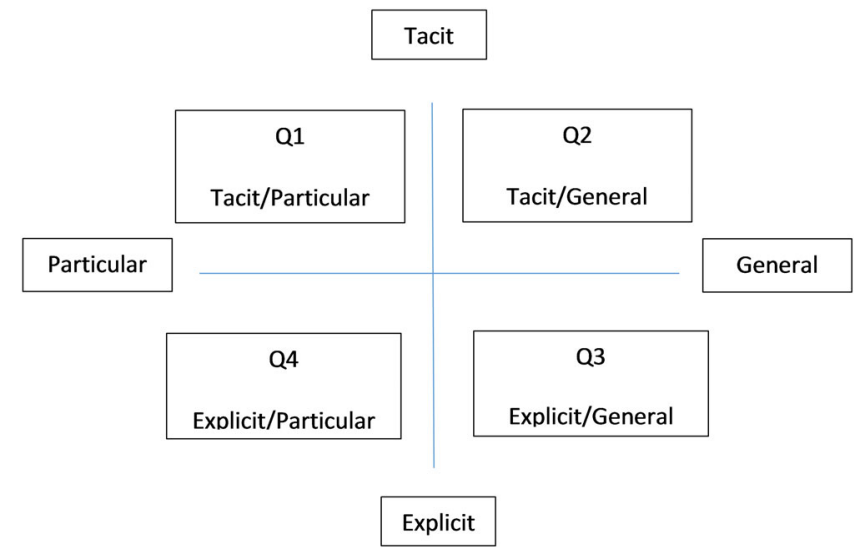

FIGURE 1 McHugh and Walker's four kinds of knowledge

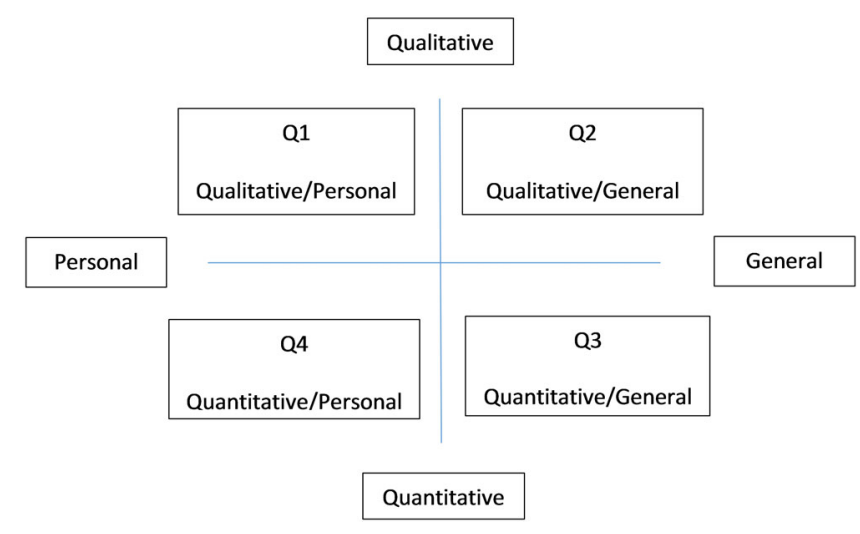

FIGURE 2 Upshur et al's four kinds of evidence

evidence (eg, Hutchinson and Rogers ${ }^{5}$ ). Also see Bluhm ${ }^{6}$ who argued that the idea of a hierarchy of evidence (which she points out is really a hierarchy of methodology) should be replaced by a "network" model that takes into account the relationship between evidence drawn from various sources, or types of studies, that is, choose whatever combination of Q1-Q4 evidence is appropriate to the question in hand.

This more inclusive view of knowledge promises to be useful in a variety of contexts. The one detailed by McHugh and Walker is based on the recognition that scientism (EBM) excludes tacit and particular knowledge (Q1 and Q2), thereby distorting clinical reality and impairing medical practice. As stated by them,

Medicine has been informed by scientific principles throughout its history. However, perhaps partly because of the success of modern medical science, scientific knowledge is often elevated above other forms of knowledge, such that it might be expected that all medical knowledge should be scientifically justified. This expectation is reinforced by the widespread adoption of 'evidence based medicine' ... whereby the idea of 'best practice' is governed primarily by the results of scientific research (pp. 583-4).
We agree, and are confident that most readers of the Journal of Evaluation in Clinical Practice share this sentiment. However, we think that it is important to recognize that the scheme set out in Figure 1 is closely related to one developed by Upshur et al (2001) (hereafter, the UVG model), and we want to emphasize the fact that the utility of this formulation is not limited to demonstrations of scientism. Thus, we go beyond recognizing Upshur et al's priority to indicating other contexts within which their model can be used to illuminate matters. We begin by detailing the UVG model of evidence.

\section{2 | THE MODEL OF EVIDENCE IN HEALTH CARE PROPOSED BY UPSHUR ET AL (2001)}

This model incorporates the four distinct but related types of evidence: qualitative/personal, qualitative/general, quantitative/general, and quantitative/personal. The model can be depicted as in Figure 2.

Slightly different descriptors are attached to the dimensions by McHugh and Walker, but the apparent differences between the models shown in Table 1 are more stylistic, than substantive. One need only identify qualitative with tacit; personal with particular; and quantitative with explicit. Upshur et al give examples of each of the four types of evidence included in their scheme. Qualitative/ personal (Q1) evidence includes that pertaining to the beliefs, attitudes, preferences, and perceptions of both the health care provider and the patient as obtained, for example, from case histories, clinical encounters, and interviews. Qualitative/general (Q2) evidence is primarily social and historical and might result from policy studies, or consensus methods like Delphi group approaches. This quadrant is necessary to capture the cultural, social, and gender dimensions of evidence. Thus, whether we think of personal views and preferences, or social views and preferences, we are clearly dealing with tacit knowledge.

The quantitative/personal (Q4) is typified by quality of life (QoL) scales; the quantitative/general (Q3) by the kinds of evidence available from EBM, in particular from the RCT. The knowledge to be gained from these sources of evidence is clearly of the explicit kind in that it is generally numerical and therefore easy to articulate.

A more important difference needs some unpacking: Upshur speaks of "evidence," McHugh of "knowledge." It is possible to see the two as but opposite sides of the same coin: knowledge is justified belief, and one's belief is justified to the extent that $s /$ he has good (relevant) evidence to support it. According to this view, the UVG model is essentially equivalent to the MW model in that UVG evidence leads to MW knowledge. As an example, a person's QoL is explicit/particular knowledge based on quantitative/personal evidence.

Alternatively, and better, the two models can be kept separate, and viewed as complementary to one another. In this formulation, UVG evidence would lead to MW knowledge only if it were both reliable and relevant to the problem in hand. This has the advantage that it emphasizes that context modifies the relevance of evidence (good evidence is not always relevant) and the complementary model 
TAB LE 1 Comparison of Upshur et al's evidence types with McHugh and Walker's kinds of knowledge

\begin{tabular}{lll} 
& Upshur et al (2000) & McHugh and Walker (2015) \\
\hline Q1 & Qualitative-personal & Tacit-particular \\
Q2 & Qualitative-general & Tacit-general \\
Q3 & Quantitative-general & Explicit-general \\
Q4 & Quantitative-personal & Explicit-particular \\
\hline
\end{tabular}

highlights this relationship as inherent to medical research and practice. This view also facilitates an appreciation of the case studies considered below. In any case, we denote by the UVGMW model the melding of the UVG and MW models. They are seen as both bringing something to the table, ingredients that are complementary (not competing!) to one another. This model is used in the following examples to illustrate the point that all four quadrants of the model are required to adequately address the questions posed.

\section{3 | OTHER APPLICATIONS OF THE MODEL}

\section{1 | The research/practice distinction}

The Belmont Report (National Commission for the Protection of Human Subjects in Biomedical and Behavioral Research) ${ }^{8}$ draws a sharp distinction between clinical practice and research, stating:

It is important to distinguish between biomedical and behavioral research, on the one hand, and the practice of accepted therapy on the other...For the most part, the term "practice" refers to interventions that are designed solely to enhance the well-being of an individual patient or client and that have a reasonable expectation of success...By contrast, the term "research" denotes an activity designed to test an hypothesis, permit conclusions to be drawn, and thereby contribute to generalizable knowledge. (p. 3)

Kowalski et $\mathrm{al}^{9}$ have argued that this purported separation does more harm than good along a number of fronts. Of most interest here is that, often, the implication is that the "evidence" that can be gained from clinical practice is not really evidence at all, and that only evidence arising in research contexts counts. Otherwise stated, only Q3 evidence is real evidence, and the other quadrants have no role to play in making healthcare decisions. As noted by Bluhm and Borgerson ${ }^{10}$ "The idea that research is a systematic activity that aims to produce generalizable knowledge implies that the contrast class-clinical careis not systematic and does not lead to new knowledge" (p. 476). The UVGMW model, however, includes other forms of evidence that have been shown to assume important roles in clinical practice. Consider, for example, that a patient, $P$, with disease $D$ cannot be replaced by $D$ alone $(P \neq D)$. If we concentrate on quantitative/general evidence (EBM) to the exclusion of other types of evidence, we may achieve good management of the disease, but a less than satisfactory outcome for the patient.

\section{2 $\quad$ Reuniting MDs and nurses}

One of the unintended side effects of the ascension of scientism/ EBM has been the estrangement of the medical and nursing professions. Medicine is increasingly seen solely in terms of the diagnosis and treatment of disease. Focus on the patient is replaced by a consideration of that patient's disease, focusing in on the particular disease mechanism causing signs and symptoms in a patient and then, based on the diagnosis, treating that disease using scientific principles and best (Q3) evidence available. Nursing, on the other hand, has a much broader holistic approach, examining the impact of a diagnosis and its treatment on a patient in the context of their lives (Q2) and values (Q1 and Q4).

Consider, for example, a patient newly diagnosed with a type of cancer. With confirmation of the diagnosis, a physician would next focus on the best way to treat the specific cancer cells, given the stage and extent of the malignancy. The treatment plan would be based on the best evidence available (EBM), balancing the expected effects of some combination of chemotherapy, radiation, gene therapy, or surgery with the anticipated response of the malignancy to that therapeutic plan. A nurse would likely focus on the patient's response to this new diagnosis, the impact of the diagnosis on the patient's roles, responsibilities, values, and desired quality of life. Part of the nurse's plan will typically include helping the patient manage the signs, symptoms and effects of the treatment.

It seems clear that these approaches, implemented collaboratively, would best support the patient in obtaining optimal outcomes in accordance with patient values and preferences. While the general orientation of the two approaches is distinguishable, they are in no way competitors-they are best viewed as mutually supportive. Indeed, $\mathrm{MD} /$ nurse collaboration is generally recognized as the preferred method for obtaining optimal clinical outcomes, ${ }^{11}$ the separation reflecting more of a convenient "division of labor," than independent spheres of activity. We suggest that an even more efficacious assignment of roles and responsibilities will result by refusing to recognize the separation in the first place. After all, the beginnings of separation begin at the beginning, ingrained in the professional schools' curricula. One of the challenges for both medicine and nursing is keeping current with the changing evidence base that supports clinical practice. Studies typically report that many clinicians in both disciplines tend to practice in accordance with how they were trained, that is, basing interventions on the evidence available at the time of their clinical education. While evidence changes over time, clinicians often continue to practice based on their original training (esp. the emphasis given to basic science courses in the medical school curriculum ${ }^{12}$ ) and experience without changing their interventions to reflect the revisions and changes in evidence as a result of research. Thus, 
considerable variation exists in many clinical settings with actual care delivered sometimes not in accordance with the best evidence available.

\section{3 | Evidence and ways of knowing in psychotherapy}

In psychotherapy, tacit knowledge (Q1 and Q2) step to the forefront, and these are considered in turn below:

Q1, intuition, and creativity. Q1 evidence in the form of "intuitive knowing" is integral to the practice of (good) psychotherapy. Over the past several decades, psychotherapy has become much more formalized in an effort to provide the standardization necessary for research purposes. ${ }^{13}$ This trend has contributed to the misconception that psychotherapy can be delivered with a high degree of precision or specificity (eg, saying the "right thing" to a particular client about a particular problem) when, in practice, psychotherapy is a fundamentally intuitive, creative exercise. A therapist's ability to be creative comes from tacit, experiential knowledge that is nonconceptual and sometimes ineffable. ${ }^{14,15}$ Such knowledge cannot be acquired by reading alone.

In contrast to a set of facts that can be examined objectively, evidence in psychotherapy consists of a constructed narrative known as the client's "story." Therapists must proceed based on their intuitive recognition of what is "going on" with clients. Good therapists listen with attention to any "funny feelings" that something might be amiss. By asking questions, offering interpretations, or suggesting actions, they attempt to articulate and explore their intuition with respect to the client's perception of what is going on. Because strategies and interventions that work for one client may not work for others, the process calls for improvisation and constant refinement based on information that is perceived tacitly and used creatively. ${ }^{14}$ Much of this occurs semi-consciously, which is to say that therapeutic encounters are often marked by vague hints, incomplete thoughts, and sudden realizations that "hit you" without deliberate analysis. Interestingly, when things are going smoothly, one can function effectively as a therapist-perhaps even more effectively-by not overthinking things. In this way, Bohart ${ }^{14}$ characterized psychotherapy as an artistic endeavor.

Q2, practical knowledge, and rules of thumb. After practicing for a number of years, one learns that psychotherapy does not actually work the way it was presented in graduate school. As opposed to the hypothetico-deductive method whereby one might test hypotheses derived from structured observations of behavior, psychotherapy is not always coherent or logical, nor is it based on probabilities. For these reasons, and out of sheer necessity, therapists must operate from a tacit framework of knowledge or evidence that guides their decision-making almost automatically, based on their accumulated experience as clinicians. ${ }^{16,17}$

Psychotherapy is a practice-focused activity by nature, and thus it requires a great deal of practical knowledge in the form of heuristics (ie, general sets of principles, outlines, or guides that can be used as mental shortcuts). ${ }^{16-19}$ For example, "rules of thumb" exist for the assessment of risk (eg, the likelihood that clients may harm themselves or others), and for making rough assessments of how serious a client's condition is before conducting a lengthy diagnostic interview or psychological testing that may not be necessary. Psychotherapy is really a stepwise process of narrowing down clients' problems, and gaining an awareness of how they understand and interact with those problems. Initially, this is done with the goal of categorizing or generalizing the presenting problem into a diagnosis. Given the nuances of making an accurate diagnosis, and the dynamic and often ambiguous nature of psychotherapy (not to mention the complexity of human behavior), it is critical for therapists to simplify their work through the use of heuristics so they can make immediate judgments about how to proceed, and modify their approach as necessary.

\subsection{Understanding why nothing works}

In this subsection we briefly consider the placebo effect. There is a voluminous literature devoted to this topic, and it is not our intention to confront this. Rather, we assume that the effect exists (a Q3 question) and point out that any attempt to understand just why this is so will necessarily involve all of the Qs. Consider, for example, pain reduction (Q4) by acupuncture. An individual's response to acupuncture will necessarily involve that person's attitudes and preferences (Q1) which will be shaped to some extent by societal viewpoints (Q2), as well as properties of the encounter between the patient and acupuncturist (Q1PxQ1A). It may also have neurobiological and/or genetic (the "placebome") connections (Q3). A good, up-to-date summary is given by Greenberg ${ }^{20}$ who may be consulted for the following quotes which we offer as food for thought: "[The] placebo effect is a biological response to an act of caring; that somehow the encounter itself calls forth healing and that the more intense and focused it is, the more healing it evokes" (p. 55) ... and, "Once you start measuring the placebo effect in a quantitative way, you're transforming it to be something other than what it is" (p. 56).

\subsection{The Salk polio vaccine trial}

We have previously ${ }^{21}$ made the point that large-scale public health interventions often require evidence beyond simple efficacy, the limit of what an RCT can provide. We aim for effectiveness, not efficacy and will favor pragmatic, not explanatory trials. ${ }^{22}$

One such summary of the sorts of evidence required is the REAIM set of criteria, as discussed in Kowalski and Mrdjenovich. ${ }^{21,23}$ The RE and AIM pieces are both part of the same study, but RE differs from AIM in that RE refers to the results available at the study's conclusion, whereas AIM refers to what happens after the study proper has been concluded. RE focuses on efficacy; AIM has to do with effectiveness. The ingredients of the RE-AIM casserole are discussed in turn below. Each is introduced by asking a question relevant to the Salk trial. 
R: Reach. In terms of the Salk trial, R asks the question of whether those agreeing to participate will adequately represent the target population. Reach is an individual-level measure of participation referring to the percentage (Q4) and characteristics (Q1) of persons included in the study.

E: Efficacy. Does the Salk vaccine work? Efficacy outcomes should include both positive and negative measures; and behavioral, quality of life (Q4), and participant satisfaction (Q1) outcomes, as well as physiologic endpoints (Q3).

A: Adoption. If the Salk vaccine is shown to be effective, will it actually be used by the target population?

I: Implementation. If the Salk vaccine is shown to be effective, can it be delivered as intended to the entire target population?

M: Maintenance. If the Salk vaccine is shown to be effective, will it remain so when delivered to the (much larger) target population?

Each of the AIM components involves Q2 evidence/knowledge. For example, Adoption clearly depends on general societal attitudes concerning vaccination. Other such attitudes that come immediately to mind include feelings about abortion, blood transfusions, euthanasia, Obamacare, and socialized medicine. Imagine the differences in testing smoking cessation programs in the 1950s (when large numbers smoked, and few wanted to stop) and currently (when relatively few smoke, but those that do and want to stop are likely to be highly motivated). Implementation and maintenance are more practical matters, for example, are any changes in delivery necessary given that the intervention will be given to large numbers of people? Can quality control measures appropriate for trial execution (sufficient for the smaller sample) be generalized to cover the target population? Such questions cannot be answered by only looking at Q3 evidence; and were in fact problematic in the Salk trial. Not all of the vaccines manufactured immediately after the announcement of the vaccine's success met minimal safety and potency standards. For example, some lots of the vaccine produced by the Cutter and Wyeth pharmaceutical company were insufficiently inactivated leading to live polio virus in more than 100000 doses and causing paralysis and death in a number of children. This is a clear example of the serious harm that can occur when the RE-AIM criteria focus on the $E$ to the exclusion of the $M$ component. Scientism can be dangerous to your health.

\subsection{Antidote to quantomania}

McHugh and Walker ${ }^{1}$ have convincingly argued that limiting consideration to only explicit/general forms of knowledge (as done in EBM) distorts clinical reality and thereby impairs medical practice. The insistence that knowledge be "scientific," "objective," "numerical" has been called quantomania, a stance characterized by "What can't be counted, simply doesn't count." It is worth pointing out that quantomania can and does distort other forms on inquiry, not only that of clinical medicine. Muller ${ }^{24}$ has detailed how "metric fixation" can have deleterious consequences in such diverse areas as the military, business and finance, and philanthropy and foreign aid.
To say that numbers should not play an exclusive role in clinical decision making is, of course, far from saying that they should play no role. Quantification will often prove an insightful maneuver. Its proper role depends on context. Our final thought is nicely summarized in a recent book by Hans Rosling, Factfulness. ${ }^{25}$ Bill Gates has been quoted as saying that this is "One of the most important books I've ever read-an indispensable guide to thinking about the world."

There are indeed a number of important guides to thinking included in the book; one that gets repeated mention, in a number of contexts, is the idea that "The world cannot be understood without numbers. But the world cannot be understood by numbers alone."

From Rosling (p. 201):

The world cannot be understood without numbers, nor through numbers alone. A country cannot function without a government, but the government cannot solve every problem. Neither the public sector nor the private sector is always the answer. No single measure of a good society can drive every aspect of its development. It's not either/or. It's both and it's case by case.

Context matters. The "case-by-case" limitation imposed by Rosling is another way of saying "Horses for courses," that it's wise to bet on the competing alterative that best matches the task in hand.

\section{4 | CONCLUSIONS}

We have in all of our work, emphasized the importance of "horses for courses," that is, that one should choose the best tool for the job in hand. Context matters. The UVGMW model is especially useful in this regard in that it that it shows how context modifies the relevance of evidence. In particular, it allows us to match the design of a study to the kind(s) of evidence required to answer the question in hand. Good evidence is not always relevant.

The total UVGMW model is meant to be general-to encompass all of medical epistemology. Asking a question may limit interest to one or another, or several, of the quadrants. Some questions will require that all four be considered. We believe that seldom, if ever, will Q3 alone (scientism) be up to the task, and this has been illustrated in several particular cases above. Matching the Qs to the question asked is part of the art of research (as is asking a good question to begin with). There is no model for this ... In the vernacular, "There is no app for that."

\section{CONFLICT OF INTEREST}

The authors declare no conflict of interest.

\section{ORCID}

Charles J. Kowalski (DD https://orcid.org/0000-0002-9534-4505

Adam J. Mrdjenovich (D) https://orcid.org/0000-0001-8794-7149 


\section{REFERENCES}

1. McHugh HM, Walker ST. "Personal knowledge" in medicine and the epistemic shortcomings of scientism. J Bioeth Inq. 2015;12:577-585.

2. Polyani M. Personal Knowledge: Towards a Post-critical Philosophy. Chicago: University of Chicago Press; 1958.

3. Henry SG. Recognizing tacit knowledge in medical epistemology. Theor Med Bioeth. 2006;27(3):187-213.

4. Henry SG. Polyani's tacit knowing and the relevance of epistemology to clinical medicine. J Eval Clin Pract. 2010;16(2):292-297.

5. Hutchinson KJ, Rogers WA. Challenging the epistemological foundations of EBM: what kind of knowledge does clinical practice require? J Eval Clin Pract. 2012;18:984-991.

6. Bluhm R. From hierarchy to network: a richer view of evidence for evidence-based medicine. Perspect Biol Med. 2005;48(3):535-547.

7. Upshur REG, VanDenKerkof EG, Goel V. Meaning and measurement: an inclusive model of evidence in health care. J Eval Clin Pract. 2001;7 (2):91-96.

8. National Commission for the Protection of Human Subjects in Biomedical and Behavioral Research. The Belmont Report US Department of Health, Education and Welfare, Bethesda, MD; 1979.

9. Kowalski CJ, Hutchinson R, Mrdjenovich AJ. The ethics of clinical care and the ethics of clinical research: Yin and Yang. J Med Philos. 2017; 42:7-32.

10. Bluhm R, Borgerson K. An epistemic argument for research-practice integration in medicine. J Med Philos. 2018;43:469-484.

11. Djulbegovic B, Guyatt GH. Progresss in evidence-based medicine: a quarter century on. Lancet. 2017;390(10092):415-423.

12. Doran GA. Scientism vs humanism in medical education. Soc Sci Med. 1983;17(23):1832-1835.

13. American Psychological Association. Presidential task force. "Evidence-based practice in psychology". Am Psychol. 2006;61(4): 271-285.

14. Bohart AC. Intuition and creativity in psychotherapy. J Construct Psychol. 1999;12(4):287-311.
15. Dean RG. Ways of knowing in clinical practice. Clin Soc Work J. 1989; 17(2):116-127.

16. Dowd ET. What changes in cognitive therapy? The role of tacit knowledge structures. J Cognit Behav Psychol. 2006;6(2):141-148.

17. O'Hara D. Reconciling technical and practical knowledge in psychotherapy through Polanyi's tacit knowing. Counsel Psychol Rev. 2012; 27(1):64-72.

18. André $M$, Borgquist L, Foldevi M, Mölstad S. Asking for "rules of thumb": a way to discover tacit knowledge in general practice. Fam Pract. 2002;19(6):617-622.

19. André M, Borgquist L, Mölstad S. Use of rules of thumb in the consultation in general practice - an act of balance between the individual and the general perspective. Fam Pract. 2003;20(5):1-9.

20. Greenberg G. Why nothing works. N Y Times Mag. November 11, 2018, 50-56.

21. Kowalski CJ, Mrdjenovich AJ. Schemata, CONSORT, and the Salk polio vaccine trial. J Med Philos. 2018;43:64-82.

22. Kowalski CJ. Pragmatic problems with clinical equipoise. Perspect Biol Med. 2010;53(2):161-173.

23. Kowalski CJ, Mrdjenovich AJ. Studying group behavior: cluster randomized trials. Am J Clin Exp Med. 2013;1(1):5-15.

24. Muller JZ. The Tyranny of Metrics. Princeton, NJ: Princeton University Press; 2018.

25. Rosling H. Factfulness: Ten Reasons You're Wrong About the World - And Why Things Are Better Than You Think. New York: Flatiron Books; 2018.

How to cite this article: Kowalski CJ, Mrdjenovich AJ, Redman RW. Scientism recognizes evidence only of the quantitative/general variety. J Eval Clin Pract. 2020;26: 452-457. https://doi.org/10.1111/jep.13330 